\title{
Pre-diagnostic statin use, lymph node status and mortality in women with stages I-III breast cancer
}

\author{
Amelia Smith $^{*},{ }^{\text {, Laura Murphy }}{ }^{2}$, Lina Zgaga ${ }^{3}$, Thomas I Barron ${ }^{1}$ and Kathleen Bennett ${ }^{2}$ \\ ${ }^{1}$ Department of Pharmacology and Therapeutics, Trinity Centre for Health Sciences, Trinity College, University of Dublin, Dublin \\ D08 W9RT, Ireland; ${ }^{2}$ Statistical and Pharmcoepidemiology Research Group, Population Health Sciences Division, Royal College of \\ Surgeons in Ireland, Dublin D02 DH60, Ireland and ${ }^{3}$ Discipline of Public Health and Primary Care, Institute of Population Health, \\ Trinity College, University of Dublin, Dublin D24 DH74, Ireland
}

Background: Recent meta-analyses suggest that pre-diagnostic statin use is associated with reduced breast cancer-specific mortality. Studies have shown that high breast tumour expression of the statin target (3-hydroxy-3-methylglutaryl coenzyme-A reductase) is associated with lymph-node negative cancer. Therefore, we examined the association between pre-diagnostic statin use and; lymph node status, breast cancer-specific and all-cause mortality.

Methods: Women with stages I-III breast cancer were identified from the National Cancer Registry of Ireland $(N=6314)$. Pre-diagnostic statin users were identified from linked prescription claims data $(N=2082)$. Relative risks were estimated for associations between pre-diagnostic statin use and lymph node status. Hazard ratios (HR) were estimated for associations between pre-diagnostic statin use and breast cancer-specific and all-cause mortality.

Results: Pre-diagnostic statin use was not associated with lymph node negative status at diagnosis. In multivariate analyses, prediagnostic statin use was associated with reduced all-cause (HR $0.7895 \%$ confidence interval $(\mathrm{Cl}) 0.69,0.89$ ) and breast cancerspecific mortality $(\mathrm{HR} 0.8195 \% \mathrm{Cl} 0.68,0.96)$. This reduction in cancer-specific mortality was greatest in statin-users with oestrogen (ER) receptor-positive tumours (HR $0.6995 \% \mathrm{Cl} 0.55,0.85$ ).

Conclusion: Patients with pre-diagnostic statin exposure had a significant reduction in breast cancer-specific mortality, which was even more pronounced in women with ER + tumours.

Statins are widely used for the prevention of cardiovascular disease through reduction of serum cholesterol (Holmes and Chen, 2012). Up to $30 \%$ of Americans over the age of 40 receive statins, and utilisation is similar across Europe (Walley et al, 2004; Robinson and Booth, 2010). Statins bind to 3-hydroxy-3-methylglutaryl coenzyme-A reductase (HMGCR); inhibiting the rate-limiting step of the cholesterol biosynthesis pathway, leading to reduced levels of mevalonate and downstream products (Tobert, 2003). Potentially anti-cancer effects of statins involve the reduction of these downstream products, which have important roles in cellular processes such as membrane integrity, protein synthesis, and cell signalling (Chan et al, 2003; Jakobisiak and Golab, 2003). In addition, a recent study suggests that statin treatment may have breast tumour anti-proliferative properties due to effects on cell cycle regulators P21 and P27 (Feldt et al, 2015). A windowof-opportunity study has shown that treatment of breast cancer patients with short duration, high-dose atorvastatin (80 mg per day) results in decreased tumour proliferation and an increase in tumour HMGCR expression (Bjarnadottir et al, 2013). Interestingly, Brennan et al (2011) found that breast cancer

*Correspondence: A Smith; E-mail: smitha25@tcd.ie

Received 7 March 2017; revised 21 June 2017; accepted 22 June 2017; published online 18 July 2017

(C) 2017 Cancer Research UK. All rights reserved 0007 - 0920/17 
patients with high-tumour HMGCR expression were more likely to have smaller, node negative cancer. However, this study did not record information on prescribed medications in these patients, and could not assess the potential effect of statin use.

A recent meta-analysis suggests that pre-diagnostic statin use is associated with significantly improved cancer-specific (HR 0.73 95\% CI 0.61, 0.89) survival in women with breast cancer (Zhong et al, 2015). In a study by Ahern et al (2011) statin use was associated with reduced breast cancer recurrence; this benefit was observed only in women with ER + tumours (HR 0.69, $95 \%$ CI $0.55,0.88$ ) and not in women with ER- tumours (HR 0.75 95\% 0.47, 1.2) (Ahern et al, 2011). This effect modification by ER status has not yet been observed in studies investigating statin exposure and breast cancer-specific survival (Mc Menamin et al, 2016).

In our study, we investigate associations between prediagnostic statin use and: (i) lymph node status at diagnosis; (ii) breast cancer-specific and all-cause mortality; and (iii) whether any associations were modified by oestrogen (ER) receptor status, in a cohort of Irish women with newly diagnosed breast cancer.

\section{METHODS}

Setting and data sources. This cohort study was carried out using records from the National Cancer Registry Ireland (NCRI), which are linked to individual-level prescription dispensing data from Ireland's Primary Care Reimbursement Services (PCRS) pharmacy claims database, as described previously (Barron et al, 2014). Information on date and cause of death are obtained from linkage to death certificates. The completeness of cancer registration is estimated to be at least 97\% (Data Quality and Completeness at the Irish National Cancer Registry, 2012). The use for research of anonymised data held by the NCRI is covered by the Health (Provision of Information) Act 1997.

The PCRS is responsible for reimbursement of dispensed medication claims made under the General Medical Services (GMS) scheme. The GMS scheme provides subsidised healthcare, including prescription medications at no/minimal cost, to approximately one third of the Irish population. Eligibility for the scheme is assessed by a combination of age and means test; therefore the data set may have an overrepresentation of older people and those with lower socioeconomic status. The PCRS database records details, including quantity and dose, of prescription drugs dispensed to patients availing of the GMS scheme. This includes all statins, which are prescription-only. Drugs are coded according to WHO-ATC classifications (WHOCC-ATC/DDD Index).

Cohort and exposure definitions. The study population comprised of women diagnosed with stages I-III breast cancer (ICD-10 C50) between 1 January 2001 and 31 December 2011. Women were included in the study population if they were aged 50-80 years at diagnosis; had GMS coverage from at least 1 year before diagnosis; and no history of invasive cancer, other than nonmelanoma skin cancer. The study population was restricted by age because younger women are less likely to be prescribed statins and older women are less likely to receive definitive cancer staging/ treatment (Hillner et al, 1996).

We identified pre-diagnostic statin prescriptions dispensed to the women in the study cohort from the PCRS database using WHO-ATC classifications (Supplementary Table 1). For each day of follow-up, we calculated statin dosing intensity from the number of days' supply of statin received in the prior year (Peterson et al, 2007). These statin exposure histories were used to define the following time-varying exposure categories: (i) women were identified as exposed (yes/no) from the date they received their first statin prescription; (ii) women were identified as having highintensity exposure once they had taken a statin at an intensity of $\geqslant 80 \%$, for at least 1 year (e.g., a statin supply for at least 292 out of a 365 day period was considered high intensity; Supplementary Figure 1). The overall intensity of statin exposure while on treatment was calculated by expressing the number of days' supply received as a proportion of the number of days from initiation to last exposure. Once allocated to an exposure category, women remained in this category to the end of follow-up. Patients with de-novo post-diagnostic statin use were excluded from analyses, so as to determine the effect of statin use in patients with pre-diagnostic use.

Covariates and outcomes. The NCRI database was used to identify lymph node status at diagnosis (positive and negative). Women were lymph node positive if they had a nodal status of N1/ $2 / 3$. The following information was also obtained from the NCRI database: age (years) at diagnosis, smoking status at diagnosis (never, past, current and unspecified), tumour presentation (organised screening, opportunistic screening, incidental, symptomatic and unknown), tumour size (T1, T2, T3 and T4), tumour stage (I, IIa, IIb, IIIa and IIIb-c), histologic tumour grade (low, intermediate, high and unspecified), ER, progesterone (PR), human epidermal growth factor-2 (HER-2) receptor status (positive, negative and unspecified) and receipt of chemotherapy (yes, no) in the year after diagnosis. Anti-ER therapy started in the year after breast cancer diagnosis (yes, no) was identified using the PCRS database (WHO-ATC classifications-Supplementary Table 1). The PCRS database was also used to identify other potentially confounding medication use in the year before diagnosis (exposed, unexposed); aspirin (Holmes et al, 2010), anti-diabetics, (Holmes et al, 2010) non-steroidal anti-inflammatory drugs (Marshall et al, 2005) and bisphosphonates (Coleman et al, 2013). The number of drug classes (fourth level WHO-ATC classification) dispensed in the year before diagnosis was used as a proxy measure of comorbidity (Schneeweiss et al, 2001). Death certificates were used to determine the date and cause of death. Breast cancer-specific deaths were identified using SEER definitions (Supplementary Table 1; Howlader et al, 2010).

Statistical analysis. The proportion of statin-users and non-users was tabulated for each covariate and differences in the rates of statin use across covariates were compared using univariate Poisson regression. Univariate and multivariate log-binomial models were used to estimate relative risks (RR) and 95\% confidence intervals (CI's) for associations between prediagnostic statin use and lymph node negative breast cancer at diagnosis.

In survival analyses, multivariable Cox proportional hazards models were used to estimate adjusted hazard ratios (HR) and 95\% CI's for associations between pre-diagnostic statin use and breast cancer-specific and all-cause mortality. Women were categorised as statin exposed (yes/no) from the time they received their first statin prescription. These exposures were lagged by 1 year in survival analyses to reduce the possibility that changes in breast cancer prognosis or treatment, for example a breast cancer recurrence or approaching death, influenced a patient's or prescriber's decision to initiate or continue statin therapy (Chubak et al, 2013; Smith et al, 2017). The previously described covariates were selected for inclusion in multivariable analyses, based on prior knowledge of patient and clinical characteristics associated with breast cancerspecific mortality.

The following pre-planned subgroup analyses were applied to both lymph-node status analyses and survival analyses. Firstly, analyses were stratified by ER status (positive, negative, unspecified). In survival analyses, the presence of effect modification by ER status was assessed with the inclusion of an interaction term in the 
multivariable model. Secondly, as prior studies have suggested that only lipophilic statin use is associated with improved breast cancer outcomes (Ahern et al, 2011) analyses were also stratified by statin solubility: lipophilic (atorvastatin, fluvastatin, simvastatin), hydrophilic (pravastatin, rosuvastatin), both (Gazzerro et al, 2012). Finally, we stratified analyses by high/low exposure intensity.

We conducted the following sensitivity analyses: (i) associations between pre-diagnostic statin use and lymph node status, all-cause and cancer-specific mortality were assessed with stratification by mode of tumour presentation; (ii) in survival analyses, high-intensity statin exposure was defined as $\geqslant 80 \%$ intensity for longer than two consecutive years; and (iii) in survival analyses, statin exposure lag time was varied ( 0,6 months, 2 years) to account for possible reverse causation bias, as mentioned above. All analyses were performed using SAS v9.3 (SAS Institute Inc, Cary, NC, USA). Results were regarded as significant at a two-sided $\alpha$-level of 0.05 .

\section{RESULTS}

Cohort and exposure characteristics. We identified 6314 women eligible for inclusion in the study (Figure 1). The characteristics of pre-diagnostic statin-users $(n=2082)$ and non-users $(n=4232)$ are presented in Table 1 . Statin-users were significantly older and had a significantly higher comorbidity score than non-users. Statin-users were also significantly more likely to be prescribed aspirin, NSAIDs, anti-diabetics and bisphosphonates.

Pre-diagnostic statin use and lymph node status. Relative risks for associations between pre-diagnostic statin use and lymph node negative breast cancer are presented in Table 2. The proportion of

Women of any age with National Cancer Registry Ireland database record of invasive breast cancer, diagnosed 1 January 2001-31 December 2011, and General Medical Services eligibility starting at least 1 year prior to diagnosis. Excluding women with prior invasive cancer ${ }^{\star}$, or breast cancer identified at death. $N=10319$

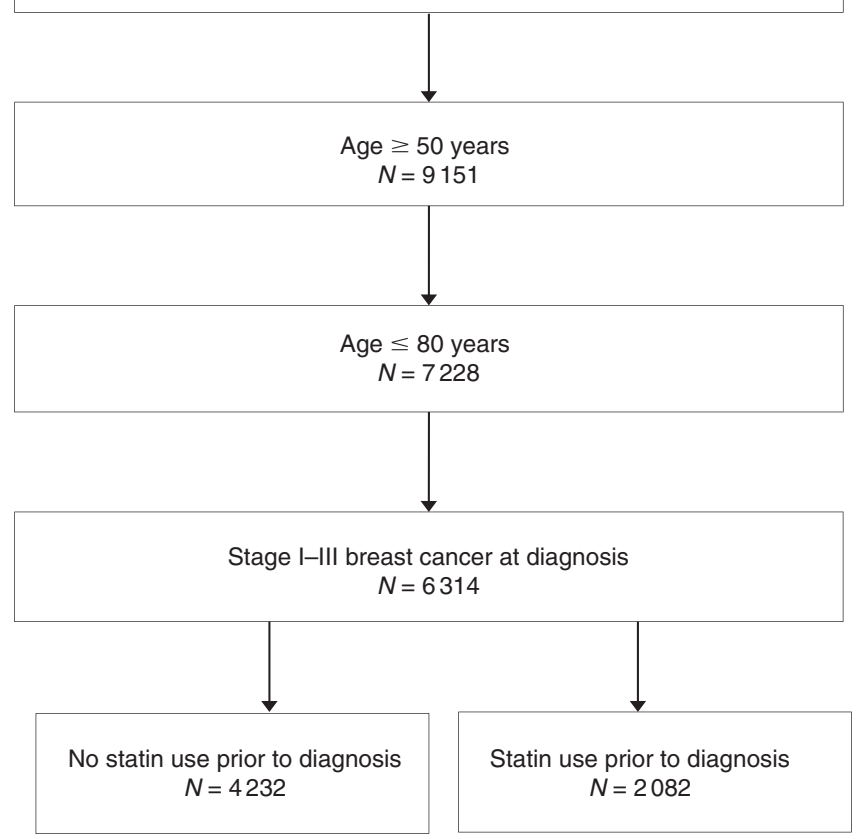

Figure 1. Flowchart for study cohort inclusion and exclusion criteria. *With the exception of non-melanoma skin cancer. women with node-negative status in the statin-user and non-user groups was $54 \%$ and $53 \%$, respectively. No significant association was found between pre-diagnostic statin use and lymph node negative status at diagnosis, in both univariate (RR $1.0195 \%$ CI $0.96,1.06$ ) and multivariate adjusted analyses (RR $1.0095 \% 0.98$, 1.03; Table 2). Analyses stratified by; high-intensity statin use, duration of statin use, and type of statin received, also yielded null

Table 1. Characteristics of women selected for inclusion in study cohort

\begin{tabular}{|c|c|c|}
\hline \multirow{2}{*}{ Characteristic } & \multicolumn{2}{|c|}{ Statin use before diagnosis } \\
\hline & $\begin{array}{l}\text { Non-user } \\
N=4232\end{array}$ & $\begin{array}{c}\text { User } \\
N=2082\end{array}$ \\
\hline \multicolumn{3}{|l|}{ Age in years ${ }^{a}$} \\
\hline Median (IQR) & $67(58,74)$ & $71(63,75)$ \\
\hline \multicolumn{3}{|l|}{ Comorbidity score ${ }^{a}$} \\
\hline Median (IQR) & $7(3,11)$ & $11(7,16)$ \\
\hline \multicolumn{3}{|l|}{ Smoking (\%) } \\
\hline Current & $885(20.9)$ & $381(18.3)$ \\
\hline Past & $490(11.6)$ & $262(12.6)$ \\
\hline Never & 2009 (47.5) & $994(47.7)$ \\
\hline Unspecified & $848(20.0)$ & $445(21.4)$ \\
\hline \multicolumn{3}{|c|}{ Tumour presentation (\%) } \\
\hline Screening; organised & $750(17.7)$ & $324(15.6)$ \\
\hline Screening; opportunistic & $51(1.2)$ & $28(1.3)$ \\
\hline Screening; unspecified & $151(3.8)$ & $86(4.1)$ \\
\hline Incidental & $87(2.1)$ & $46(2.2)$ \\
\hline Symptomatic & $2990(70.7)$ & $1476(70.9)$ \\
\hline Unspecified & $203(4.8)$ & $122(5.9)$ \\
\hline \multicolumn{3}{|c|}{ Tumour morphology (\%) } \\
\hline Lobular & $527(12.5)$ & $273(13.1)$ \\
\hline Ductal & 3098 (73.2) & $1543(74.1)$ \\
\hline Other & $607(14.3)$ & $266(12.8)$ \\
\hline \multicolumn{3}{|l|}{ Aspirin (\%) ${ }^{a}$} \\
\hline Yes & 713 (16.9) & $1061(51.0)$ \\
\hline No & $3519(83.1)$ & $1021(49.0)$ \\
\hline \multicolumn{3}{|l|}{ NSAID (\%) ${ }^{a}$} \\
\hline Yes & $1848(43.7)$ & $988(47.5)$ \\
\hline No & $2384(56.3)$ & $1094(52.5)$ \\
\hline \multicolumn{3}{|l|}{ Anti-diabetic (\%) ${ }^{a}$} \\
\hline Yes & $143(3.4)$ & $330(15.9)$ \\
\hline No & $4089(96.6)$ & $1752(84.1)$ \\
\hline \multicolumn{3}{|l|}{ Chemotherapy (\%) ${ }^{a, b}$} \\
\hline Yes & $1685(39.8)$ & $718(34.5)$ \\
\hline No & $2547(60.2)$ & $1364(65.5)$ \\
\hline \multicolumn{3}{|l|}{ Anti-ER $(\%)^{a, b}$} \\
\hline Yes & $3131(74.0)$ & $1630(78.3)$ \\
\hline No & $1101(26.0)$ & $452(21.7)$ \\
\hline \multicolumn{3}{|l|}{ Bisphosphonate (\%) ${ }^{a}$} \\
\hline Yes & $326(7.7)$ & $283(13.6)$ \\
\hline No & $3906(92.3)$ & $1799(86.4)$ \\
\hline \multicolumn{3}{|l|}{ Nodal status (\%) } \\
\hline Positive & $1756(41.7)$ & $847(40.7)$ \\
\hline Negative & 2261 (53.4) & $1125(54.0)$ \\
\hline Unspecified & $215(5.1)$ & $110(5.3)$ \\
\hline \multicolumn{3}{|l|}{ Tumour size (\%) } \\
\hline TO & $31(0.7)$ & $18(0.9)$ \\
\hline T1 & $1796(42.4)$ & $907(43.6)$ \\
\hline T2 & $1850(43.7)$ & $919(44.1)$ \\
\hline T3 & $262(6.2)$ & $134(6.4)$ \\
\hline T4 & $283(6.7)$ & $98(4.7)$ \\
\hline Unspecified & $10(0.2)$ & $6(0.3)$ \\
\hline
\end{tabular}




\begin{tabular}{|c|c|c|}
\hline \multirow[b]{2}{*}{ Characteristic } & \multicolumn{2}{|c|}{ Statin use before diagnosis } \\
\hline & $\begin{array}{l}\text { Non-user } \\
N=4232\end{array}$ & $\begin{array}{c}\text { User } \\
N=2082\end{array}$ \\
\hline \multicolumn{3}{|c|}{ Tumour stage $(\%)^{a}$} \\
\hline I & $1366(32.3)$ & $687(33.0)$ \\
\hline Ila & 1333 (31.5) & 675 (32.4) \\
\hline llb & $882(20.8)$ & $428(20.6)$ \\
\hline IIla & $263(6.2)$ & $140(6.7)$ \\
\hline Illb-c & $388(9.2)$ & $152(7.3)$ \\
\hline \multicolumn{3}{|c|}{ Tumour grade $(\%)^{a}$} \\
\hline Low & $454(10.7)$ & 201 (9.7) \\
\hline Intermediate & $2079(49.1)$ & $1087(52.2)$ \\
\hline High & $1352(32.0)$ & $673(32.3)$ \\
\hline Unspecified & 347 (8.2) & $121(5.8)$ \\
\hline \multicolumn{3}{|l|}{$\operatorname{ER}(\%)^{a}$} \\
\hline Negative & $720(17.0)$ & 326 (15.7) \\
\hline Positive & $3066(72.5)$ & 1605 (77.1) \\
\hline Unspecified & $446(10.5)$ & $151(7.3)$ \\
\hline \multicolumn{3}{|l|}{ PR $(\%)^{a}$} \\
\hline Negative & $1109(26.2)$ & $534(25.7)$ \\
\hline Positive & 2108 (49.8) & $1170(56.2)$ \\
\hline Unspecified & $1015(24.0)$ & $378(18.2)$ \\
\hline \multicolumn{3}{|l|}{ HER2 $(\%)^{a}$} \\
\hline Negative & 2511 (59.3) & $1460(70.1)$ \\
\hline Positive & $530(12.5)$ & $246(11.8)$ \\
\hline Unspecified & $1191(28.1)$ & $376(18.1)$ \\
\hline \multicolumn{3}{|c|}{ 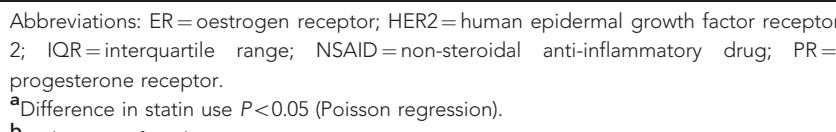 } \\
\hline
\end{tabular}

findings (Table 2). No effect modification was observed by ER status, or mode of tumour presentation (Table 2). In univariate analyses, statin-users with breast cancers diagnosed through mammography screening were significantly more likely to be lymph node negative (RR 1.32, 95\% CI 1.23, 1.43); however, this effect was non-significant in multivariable adjusted analyses (RR $1.01,95 \%$ CI $0.95,1.08$ ) (Table 2).

Pre-diagnostic statin use and mortality. After lagging statin exposure by 1 year, we identified 2024 women with prediagnostic statin use. In multivariable adjusted survival analyses, pre-diagnostic statin use was associated with a significant, 19\% reduction in breast cancer-specific mortality (HR 0.81 95\% CI 0.68 , 0.96 ) and a significant $22 \%$ reduction in all-cause mortality (HR 0.78 95\% CI 0.69, 0.89; Table 3). This cancer-specific survival benefit was observed in women with high-intensity use (HR 0.70 95\% CI 0.52, 0.94) but not in those with low-intensity statin use (HR $0.9095 \%$ CI $0.67,1.2$ ). In analyses stratified by type of statin received (hydrophilic, lipophilic, both), survival benefit was significant in women who received a lipophilic statin (HR 0.76 95\% CI 0.61, 0.95) but not hydrophilic statin.

In multivariable survival analyses stratified by mode of tumour presentation, a similar effect on all-cause (HR $0.7895 \%$ CI $0.68,0.90$ ) and breast cancer-specific (HR 0.83 95\% CI 0.68 , $1.00)$ mortality was seen in those with tumours diagnosed through symptomatic presentation. This effect was not seen in women with tumours diagnosed through organised screening; however, this may be due to fewer numbers of women in this subgroup (Table 3).

In analyses of effect-modification by ER status, pre-diagnostic statin use was associated with a more marked, statistically significant, $31 \%$ reduction in breast cancer-specific mortality in patients with ER + tumours (HR 0.69 95\% CI $0.55,0.85$ )

Table 2. Univariate and multivariate RRs for associations between pre-diagnostic statin use and lymph node negative breast cancer

Node negative breast cancer

\begin{tabular}{|c|c|c|c|c|c|c|c|c|}
\hline & Node + & (\%) & Node - & (\%) & \multicolumn{2}{|c|}{ Univariate RR $(95 \% \mathrm{Cl})$} & \multicolumn{2}{|c|}{ Multivariate RR $\left(95 \% \mathrm{Cl}^{\mathrm{a}}\right.$} \\
\hline \multicolumn{9}{|l|}{ Statin exposure } \\
\hline Non-user & 1756 & 41.5 & 2261 & 53.4 & Ref & - & Ref & - \\
\hline Prediagnostic statin-user & 847 & 40.7 & 1125 & 54.0 & 1.01 & $0.96,1.06$ & 1.00 & $0.98,1.03$ \\
\hline \multicolumn{9}{|l|}{ Hydro/lipophilic } \\
\hline Non-user & 1756 & 41.5 & 2261 & 53.4 & Ref & - & Ref & - \\
\hline Hydrophilic statin-user & 216 & 36.9 & 335 & 57.2 & 1.07 & $1.00,1.16$ & 1.00 & $0.97,1.04$ \\
\hline Lipophilic statin-user & 444 & 41.9 & 562 & 53.0 & 0.99 & $0.93,1.05$ & 1.00 & $0.97,1.03$ \\
\hline Both & 186 & 43.0 & 226 & 52.2 & 0.97 & $0.89,1.07$ & 1.01 & $0.97,1.05$ \\
\hline \multicolumn{9}{|l|}{ Dosing intensity } \\
\hline Non-user & 1756 & 41.5 & 2261 & 53.4 & Ref & - & Ref & - \\
\hline Low-intensity user & 163 & 41.3 & 204 & 51.7 & 0.96 & $0.87,1.06$ & 0.98 & $0.94,1.02$ \\
\hline High-intensity user & 684 & 40.6 & 921 & 54.6 & 1.03 & $0.97,1.09$ & 1.01 & $0.99,1.04$ \\
\hline \multicolumn{9}{|l|}{ Effect modification ER + } \\
\hline Non-user & 1756 & 41.5 & 2261 & 53.4 & Ref & - & Ref & - \\
\hline Pre-diagnostic statin-user & 636 & 39.6 & 883 & 55.0 & 1.04 & $0.98,1.09$ & 1.01 & $0.97,1.06$ \\
\hline \multicolumn{9}{|c|}{ Symptomatic presentation } \\
\hline Non-user & 1756 & 41.5 & 2261 & 53.4 & Ref & - & Ref & - \\
\hline Pre-diagnostic statin-user & 659 & 44.7 & 735 & 49.8 & 0.91 & $0.86,0.96$ & 1.00 & $0.97,1.03$ \\
\hline \multicolumn{9}{|l|}{ Screening presentation } \\
\hline Non-user & 1756 & 41.5 & 2261 & 53.4 & Ref & - & Ref & - \\
\hline Pre-diagnostic statin-user & 95 & 29.3 & 227 & 70.1 & 1.32 & $1.23,1.43$ & 1.01 & $0.95,1.08$ \\
\hline
\end{tabular}


Table 3. Univariate and multivariate hazard ratios for associations between pre-diagnostic statin use and mortality

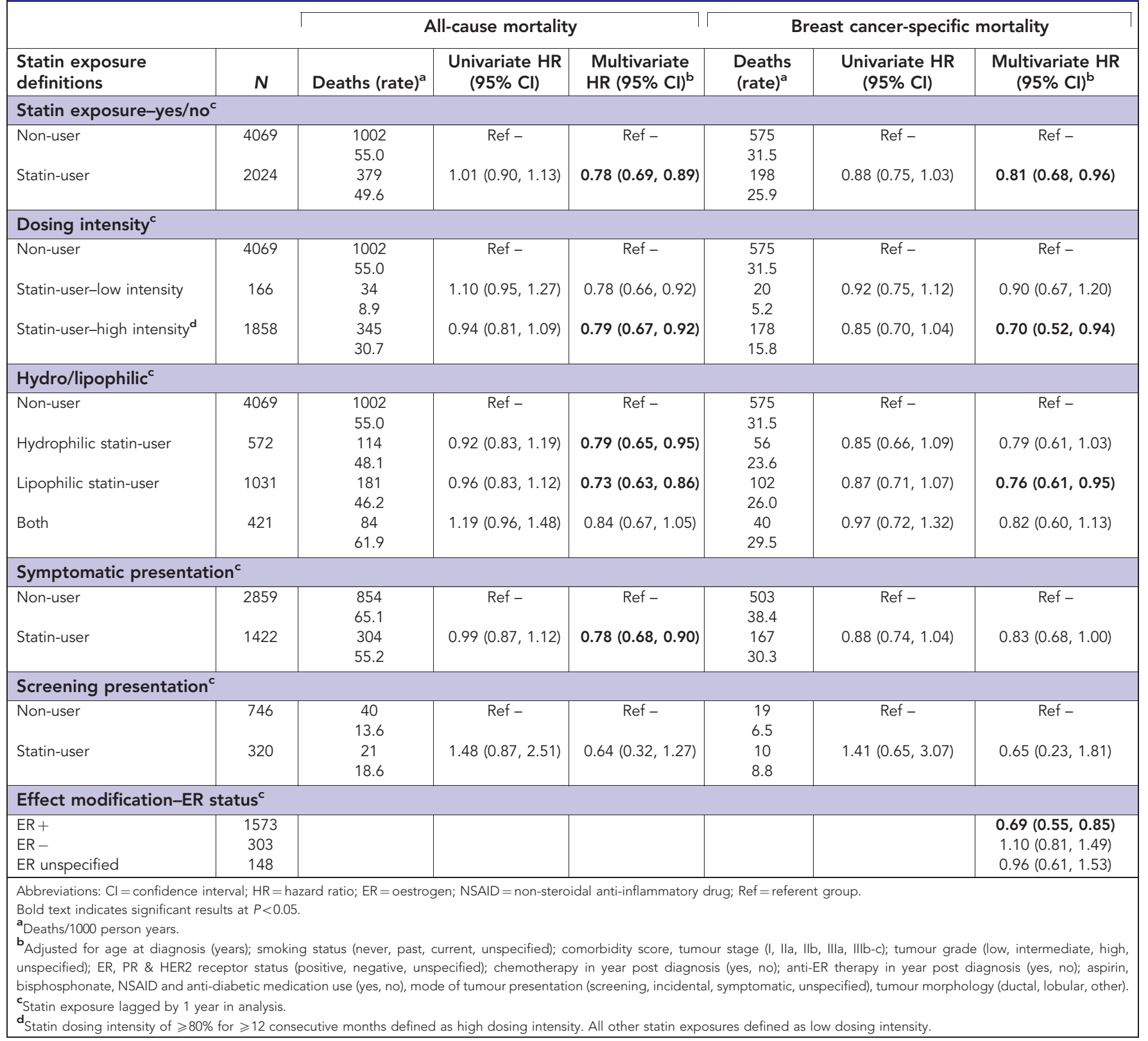

$($ Pinteraction $<0.01)$ (Table 3$)$. This survival benefit was not observed in women with ER- tumours (HR 1.10 95\% CI 0.81, 1.10; Table 3).

In sensitivity analyses, a similar reduction in breast cancerspecific mortality was observed when high-intensity exposure window was increased to 2 years (HR $0.6795 \%$ CI $0.47,0.94$ ) (Table 4). Again, a similar effect was seen when varying the statin exposure lag time in survival analyses (Table 4).

\section{DISCUSSION}

In this study of 6314 women with stage I-III breast cancer, pre-diagnostic statin use was not significantly associated with lymph node status at diagnosis but was associated with a statistically significant reduction in all-cause and breast cancer-specific mortality, even when adjusting for major prognostic factors. The survival benefit was even more pronounced in women with ER + tumours.

The survival benefit observed is similar to findings from a metaanalysis of studies investigating statin use and breast cancerspecific mortality by Zhong et al (2015) (HR 0.73, 95\% CI 0.62 , 0.86), and another by Mansourian et al (2016) (HR 0.85 95\% CI $0.83,0.87$ ) (Mansourian et al, 2016). Our study showed cancerspecific survival benefit was strongest among women receiving lipophilic statins (HR 0.76), and in those with high-intensity statin exposure (HR 0.70). The exact cause of reductions in breast cancer mortality is still largely unknown. However, possible mechanisms have been suggested; pre-clinical studies have shown effects on cell signalling through stabilisation of cyclin-dependent kinase inhibitors p21 and p27 (Denoyelle et al, 2001). Statins have also been shown to exhibit immunomodulatory properties; cerivastatin was shown to enhance tumour CD8 + T-cell infiltration and induced tumour associated macrophages to an M1-like phenotype; creating an anti-tumour environment (Mira et al, 2013). 
Table 4. Sensitivity analyses-univariate and multivariate hazard ratios for associations between statin use and mortality

\begin{tabular}{|c|c|c|c|c|c|c|c|}
\hline & & \multicolumn{3}{|c|}{ All-cause mortality } & \multicolumn{3}{|c|}{ Breast cancer-specific mortality } \\
\hline $\begin{array}{l}\text { Statin exposure } \\
\text { definitions }\end{array}$ & $N$ & $\begin{array}{l}\text { Deaths } \\
\text { (rate) }^{a}\end{array}$ & $\begin{array}{l}\text { Univariate HR } \\
\qquad(95 \% \mathrm{Cl})\end{array}$ & $\begin{array}{l}\text { Multivariate HR } \\
(95 \% \mathrm{Cl})^{\mathrm{b}}\end{array}$ & $\begin{array}{l}\text { Deaths } \\
\text { (rate) }^{a}\end{array}$ & $\begin{array}{l}\text { Univariate HR } \\
\qquad(95 \% \mathrm{Cl})\end{array}$ & $\begin{array}{l}\text { Multivariate HR } \\
\qquad(95 \% \mathrm{Cl})^{\mathrm{b}}\end{array}$ \\
\hline \multicolumn{8}{|c|}{ Sensitivity analysis: varied exposure lag times } \\
\hline \multicolumn{8}{|l|}{$\begin{array}{l}\text { Statin exposure-yes/no (lag } 0 \\
\text { years) }\end{array}$} \\
\hline Non-user & 4232 & $\begin{array}{l}1165 \\
48.1\end{array}$ & Ref - & Ref - & $\begin{array}{l}682 \\
28.2\end{array}$ & Ref - & Ref - \\
\hline Statin-user & 2082 & $\begin{array}{l}437 \\
55.5\end{array}$ & $1.01(0.92,1.13)$ & $0.77(0.68,0.87)$ & $\begin{array}{l}230 \\
29.2\end{array}$ & $0.87(0.75,1.00)$ & $0.77(0.66,0.90)$ \\
\hline \multicolumn{8}{|l|}{$\begin{array}{l}\text { Statin exposure-yes/no (lag } 6 \\
\text { months) }\end{array}$} \\
\hline Non-user & 4149 & $\begin{array}{l}1082 \\
51.1\end{array}$ & Ref - & Ref - & $\begin{array}{l}630 \\
29.7\end{array}$ & Ref - & Ref - \\
\hline Statin-user & 2052 & $\begin{array}{l}407 \\
52.5\end{array}$ & $1.00(0.90,1.12)$ & $0.77(0.68,0.88)$ & $\begin{array}{l}217 \\
28.0\end{array}$ & $0.88(0.76,1.03)$ & $0.80(0.68,0.95)$ \\
\hline \multicolumn{8}{|l|}{$\begin{array}{l}\text { Statin exposure-yes/no (lag } 1 \\
\text { year, included for reference) }\end{array}$} \\
\hline Non-user & 4069 & $\begin{array}{l}1002 \\
55.0\end{array}$ & Ref - & Ref - & $\begin{array}{l}575 \\
31.5\end{array}$ & Ref - & Ref - \\
\hline Statin-user & 2024 & $\begin{array}{l}379 \\
49.6\end{array}$ & $1.01(0.90,1.13)$ & $0.78(0.69,0.89)$ & $\begin{array}{l}198 \\
25.9\end{array}$ & $0.88(0.75,1.03)$ & $0.81(0.68,0.96)$ \\
\hline \multicolumn{8}{|c|}{ Statin exposure-yes/no (lag 2 years) } \\
\hline Non-user & 3566 & $\begin{array}{l}832 \\
58.8\end{array}$ & Ref - & Ref - & $\begin{array}{l}462 \\
32.6\end{array}$ & Ref - & Ref - \\
\hline Statin-user & 1701 & $\begin{array}{l}301 \\
50.0\end{array}$ & $1.03(0.90,1.17)$ & $0.81(0.70,0.93)$ & $\begin{array}{r}148 \\
24.5\end{array}$ & $0.87(0.73,1.04)$ & $0.81(0.66,0.98)$ \\
\hline \multicolumn{8}{|c|}{ Sensitivity analysis: high-intensity exposure $\geqslant 80 \%$ for $\geqslant 24$ consecutive months $^{c}$} \\
\hline Non-user & 4069 & 1002 & Ref - & Ref - & $\begin{array}{l}575 \\
53.1\end{array}$ & Ref - & Ref - \\
\hline Statin-user-low intensity & 302 & 64 & $1.08(0.95,1.23$ & $0.81(0.70,0.94)$ & 35 & $0.92(0.77,1.11)$ & $0.87(0.67,1.13)$ \\
\hline Statin-user-high intensity & 1722 & 315 & $0.89(0.74,1.07)$ & $0.73(0.61,0.89)$ & 163 & $0.80(0.63,1.03)$ & $0.67(0.47,0.94)$ \\
\hline \multicolumn{8}{|c|}{ 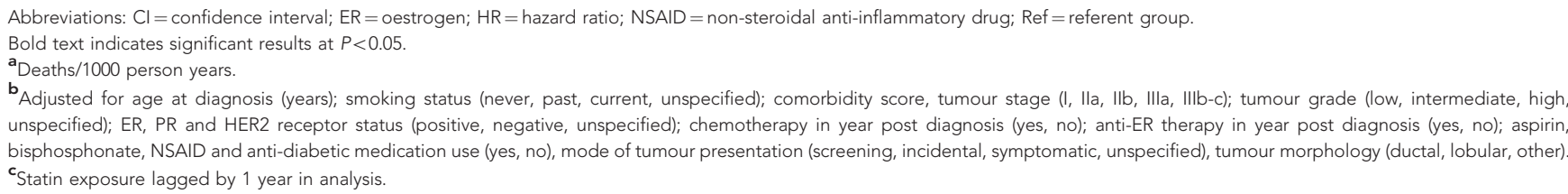 } \\
\hline
\end{tabular}

A number of studies have been published investigating associations between pre and/or post-diagnostic statin exposure and breast cancer outcomes (Kwan et al, 2008; Ahern et al, 2011; Chae et al, 2011; Brewer et al, 2013; Nickels et al, 2013; Murtola et al, 2014; Cardwell et al, 2015; Desai et al, 2015; Zhong et al, 2015; Mansourian et al, 2016; Manthravadi et al, 2016; Smith et al, 2016). To our knowledge, this is the first study investigating associations between pre-diagnostic statin use and lymph node status at diagnosis. In our study, pre-diagnostic statin use was not associated with lymph node negativity in multivariable adjusted analyses. Relative risks remained unchanged after stratification by statin type and statin intensity. In a clinical trial in which breast cancer patients were administered short-term high-dose (80 mg per day) atorvastatin; post-treatment tumour biopsies had significantly increased expression of HMGCR, the target enzyme for statins (Bjarnadottir et al, 2013). Interestingly, moderate/strong HMGCR expression in breast tumour biopsies has been shown to be associated with a less aggressive tumour phenotype; lymph node negativity, lower grade and ER/PR positivity (Gustbée et al, 2015). Although we did not observe an association between pre-diagnostic statin exposure and lymph node negativity in our study, it is possible that there may be specific subgroups of patients, for example; those with tumour expression of HMGCR, for whom statin treatment may be beneficial. In this study, we do not have access to reliable recurrence information, and it is possible that reductions in breast cancer specific mortality in statin-users are due to a reduction in cancer recurrence. Interestingly, Brennan et al (2011) showed that tumour HMGCR protein expression was associated with tamoxifen response in a cohort of over 500 women. We found a more marked reduction in breast cancer mortality for users of lipophilic statins (HR 0.76), which is in keeping with previous studies (Campbell et al, 2006; Ghosh-Choudhury et al, 2010; Ahern et al, 2011; Cardwell et al, 2015). However, it should be noted that the numbers of patients receiving a hydrophilic statin were much lower than lipophilic, and any association may be under-powered. In addition, atorvastatin is considered hydrophilic in the study by Ahern et al (2011), which is unlike the other previously mentioned studies. Studies have shown that lipophilic statins can inhibit breast cancer cell survival and cell proliferation

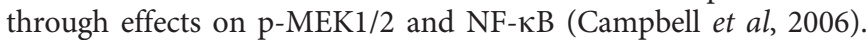
Lipophilic statins have been shown to inhibit anti-apoptotic Bcl-XL expression and induce the expression of pro-apoptotic/antiproliferative PTEN (Ghosh-Choudhury et al, 2010). In addition, lipophilic statin use was associated with reduced breast cancer recurrence in a Danish cohort of women with breast cancer (Ahern et al, 2011). A possible explanation for the differential effect by statin structure is due to lipophilic statins being more widely distributed throughout the body and their ability to penetrate the plasma membrane passively (Matusewicz et al, 2015). Hydrophilic statins, however, require uptake by the 
OATP1B1 transporter which is mainly found in the liver (Matusewicz et al, 2015).

A $30 \%$ risk reduction in breast cancer mortality was observed in women with high-intensity statin exposure. When the minimum period with high-intensity exposure was extended to 2 years in a sensitivity analysis (i.e., receiving a statin for at least 584 days in a 730 day period), the cancer-specific survival benefit was even greater (HR 0.67). This suggests a possible dose-response relationship between statin exposure and improved breast cancer survival. However, it should be noted that over $85 \%$ of statin-users were high-intensity users.

To our knowledge, this is the first study to report a significant reduction in breast cancer mortality, stratified by ER status. In a study investigating statin use and breast cancer stage, lipophilic statin-users were significantly less likely to present with late-stage breast cancer at diagnosis (HR 0.80), and this was more marked in those with ER + tumours (HR 0.72) (Desai et al, 2015). Ahern et al (2011) found that significant reductions in breast cancer recurrence in lipophilic statin-users were confined to ER + patients (HR 0.69); however, it should be noted that over $70 \%$ of women had ER+ tumours (Ahern et al, 2011). Unfortunately, we did not have access to recurrence information and cannot determine whether reductions in breast cancer mortality in our study are due to reduced recurrence in statin-users. In a recent randomised, phase III, double-blind clinical trial, concomitant cholesterol-lowering medication and aromatase inhibitors was associated with improved disease-free survival (HR $0.76,95 \%$ CI $0.60,0.97$ ) in women with early stage, hormone-receptor-positive breast cancer (Borgquist et al, 2017). It is known that 27-hydroxycholesterol $(27 \mathrm{HC})$ is cholesterol metabolite and a selective ER receptor modulator (SERM) capable of promoting proliferation in ER + cells (McDonnell et al, 2014). As statins decrease the level of cholesterol in the circulation, and subsequent level of $27 \mathrm{HC}$, it is possible that this leads to a decrease in ER + tumour cell profileration (Kimbung et al, 2016). As mentioned, tumour expression of HMGCR may have an important role in the anticancer properties of statins. Interestingly, in studies investigating the prognostic role of breast tumour HMGCR expression, a combination of both HMGCR and ER positivity was associated with improved response to tamoxifen (Brennan et al, 2011), breast cancer-specific survival and recurrence free survival (Borgquist et al, 2008). As literature accumulates, it is clear that there may be important clinical implications for statin-use and breast cancer outcomes. However, there may be specific subgroups of women which may benefit, and the complex interplay between statin exposure, HMGCR expression, ER status, and subsequent cancer outcomes warrant further investigation.

Our study has a number of strengths, including the use of accurate cancer outcome and prescription refill data. However, there are some limitations. We could not verify whether women took the medication and non-compliance may have resulted in exposure misclassification. However, we expect that women are unlikely to continue filling prescriptions for medication they no longer take. It is important to consider that statins may be preferentially prescribed for, and taken by, patients who engage in healthier behaviours and have superior health outcomes (Evans et al, 1995; Brookhart et al, 2007; Flahavan et al, 2014). This is known as healthy-user bias and may cause an overestimation of any beneficial effect of statins (Glynn et al, 2006). In our study, the rates of breast cancers detected through mammography screening were similar in statin-users and non-users, suggesting that healthy-user bias may be minimal (Brookhart et al, 2007). We did not have information on all lifestyle factors that may influence disease progression, for example, BMI, and the potential for residual confounding in our analyses should be considered. Up to $28 \%$ of women have an unspecified HER2 status; these women may have been diagnosed before the introduction of the American Society of Clinical
Oncology/College of American Pathologists (ASCO/CAP) HER2 testing guidelines in 2007 (Tchrakian et al, 2015). Finally, when generalising our results, it should be noted that our study population was a subset of breast cancer cases defined by eligibility for the GMS scheme.

To conclude, the results from our study suggest that prediagnostic statin use in women with stages I-III breast cancer is associated with a significant reduction in both breast cancerspecific and all-cause mortality, particularly in those with ER + breast cancer, but is not significantly associated with lymph node status at diagnosis. In future studies, we suggest that the association between statin exposure, tumour HMGCR expression, and breast cancer outcomes be explored further.

\section{ACKNOWLEDGEMENTS}

We thank the National Cancer Registry Ireland and the Irish Health Services Executive Primary Care Reimbursements Services for providing access to the data upon which this study was based. In particular, we are grateful to the Data Team at the National Cancer Registry Ireland for linking the data sets and Dr Sandra Deady, Mr Christopher Brown and Prof Linda Sharp for preparing these for analysis. The interpretation and reporting of these data are the responsibility of the authors and should in no way be seen as the official policy or interpretation of the National Cancer Registry Ireland or the Irish Health Services Executive Primary Care Reimbursements Services. This work was supported by the Irish Cancer Society Collaborative Cancer Research Centre BREAST-PREDICT (CCRC13GAL to KB and TIB). LM and AS positions are funded by the Irish Cancer Society Collaborative Cancer Research Centre BREAST-PREDICT (CCRC13GAL). TIB (ICE/2011/9) and KB (RLA/2015/1579) position was funded by the Health Research Board Ireland. The Health Research Board Ireland and the Irish Cancer Society had no role in the study design; collection, analysis and interpretation of the data; writing of the report; or the decision to submit for publication.

\section{CONFLICT OF INTEREST}

The authors declare no conflict of interest.

\section{REFERENCES}

Ahern TP, Pedersen L, Tarp M, Cronin-Fenton DP, Garne JP, Silliman RA, Sørensen HT, Lash TL (2011) Statin prescriptions and breast cancer recurrence risk: A Danish nationwide prospective cohort study. J Natl Cancer Inst 103: 1461-1468.

Barron TI, Flahavan EM, Sharp L, Bennett K, Visvanathan K (2014) Recent prediagnostic aspirin use, lymph node involvement, and 5-year mortality in women with stage I-III breast cancer: a nationwide population-based cohort study. Cancer Res 74: 4065-4077.

Bjarnadottir O, Romero Q, Bendahl P-O, Jirström K, Rydén L, Loman N, Uhlén M, Johannesson H, Rose C, Grabau D, Borgquist S (2013) Targeting HMG-CoA reductase with statins in a window-of-opportunity breast cancer trial. Breast Cancer Res Treat 138: 499-508.

Borgquist S, Giobbie-Hurder A, Ahern TP, Garber JE, Colleoni M, Láng I, Debled M, Ejlertsen B, von Moos R, Smith I, Coates AS, Goldhirsch A, Rabaglio M, Price KN, Gelber RD, Regan MM, Thürlimann B (2017) Cholesterol, cholesterol-lowering medication use, and breast cancer outcome in the BIG 1-98 Study. J Clin Oncol 35: 1179-1188.

Borgquist S, Jogi A, Ponten F, Ryden L, Brennan DJ, Jirstrom K (2008) Prognostic impact of tumour-specific HMG-CoA reductase expression in primary breast cancer. Breast Cancer Res BCR 10: R79. 
Brennan DJ, Laursen H, O'Connor DP, Borgquist S, Uhlen M, Gallagher WM, Pontén F, Millikan RC, Rydén L, Jirström K (2011) Tumor-specific HMG-CoA reductase expression in primary premenopausal breast cancer predicts response to tamoxifen. Breast Cancer Res BCR 13: R12.

Brewer TM, Masuda H, Liu DD, Shen Y, Liu P, Iwamoto T, Kai K, Barnett CM, Woodward WA, Reuben JM, Yang P, Hortobagyi GN, Ueno NT (2013) Statin use in primary inflammatory breast cancer: a cohort study. Br J Cancer 109: 318-324.

Brookhart MA, Patrick AR, Dormuth C, Avorn J, Shrank W, Cadarette SM, Solomon DH (2007) Adherence to lipid-lowering therapy and the use of preventive health services: an investigation of the healthy user effect. Am J Epidemiol 166: 348-354.

Campbell MJ, Esserman LJ, Zhou Y, Shoemaker M, Lobo M, Borman E, Baehner F, Kumar AS, Adduci K, Marx C, Petricoin EF, Liotta LA, Winters M, Benz S, Benz CC (2006) Breast cancer growth prevention by statins. Cancer Res 66: 8707-8714.

Cardwell CR, Hicks BM, Hughes C, Murray LJ (2015) Statin use after diagnosis of breast cancer and survival: a population-based cohort study. Epidemiol Camb Mass 26: 68-78.

Chae YK, Valsecchi ME, Kim J, Bianchi AL, Khemasuwan D, Desai A, Tester W (2011) Reduced risk of breast cancer recurrence in patients using ACE inhibitors, ARBs, and/or statins. Cancer Invest 29: 585-593.

Chan KKW, Oza AM, Siu LL (2003) The statins as anticancer agents. Clin Cancer Res 9: 10-19.

Chubak J, Boudreau DM, Wirtz HS, McKnight B, Weiss NS (2013) Threats to validity of nonrandomized studies of postdiagnosis exposures on cancer recurrence and survival. J Natl Cancer Inst 105: 1456-1462.

Coleman R, Gnant M, Paterson A, Powles T, Minckwitz G, von, Pritchard K, Bergh J, Bliss J, Gralow J, Anderson S, Evans V, Pan H, Bradley R, Davies C, Gray R (2013) Effects of bisphosphonate treatment on recurrence and cause-specific mortality in women with early breast cancer: A meta-analysis of individual patient data from randomised trials. Cancer Res 73: S4-S7.

Data quality and completeness at the Irish National Cancer Registry (2012) National Cancer Registry Ireland. Available at: http://www.ncri.ie/ publications/statistical-reports/data-quality-and-completeness-irishnational-cancer-registry.

Denoyelle C, Vasse M, Körner M, Mishal Z, Ganné F, Vannier JP, Soria J, Soria C (2001) Cerivastatin, an inhibitor of HMG-CoA reductase, inhibits the signaling pathways involved in the invasiveness and metastatic properties of highly invasive breast cancer cell lines: an in vitro study. Carcinogenesis 22: 1139-1148.

Desai P, Lehman A, Chlebowski RT, Kwan ML, Arun M, Manson JE, Lavasani S, Wasswertheil-Smoller S, Sarto GE, LeBoff M, Cauley J, Cote M, Beebe-Dimmer J, Jay A, Simon MS (2015) Statins and breast cancer stage and mortality in the Women's Health Initiative. Cancer Causes Control 26(4): 529-539.

Evans JS, Harries C, Dennis I, Dean J (1995) General practitioners' tacit and stated policies in the prescription of lipid lowering agents. $\mathrm{Br} J$ Gen Pract $J$ $R$ Coll Gen Pract 45: 15-18.

Feldt M, Bjarnadottir O, Kimbung S, Jirström K, Bendahl P-O, Veerla S, Grabau D, Hedenfalk I, Borgquist S (2015) Statin-induced antiproliferative effects via cyclin D1 and p27 in a window-of-opportunity breast cancer trial. J Transl Med 13: 133.

Flahavan EM, Drummond FJ, Bennett K, Barron TI, Sharp L (2014) Prostate specific antigen testing is associated with men's psychological and physical health and their healthcare utilisation in a nationally representative sample: a cross-sectional study. BMC Fam Pract 15: 121.

Gazzerro P, Proto MC, Gangemi G, Malfitano AM, Ciaglia E, Pisanti S, Santoro A, Laezza C, Bifulco M (2012) Pharmacological actions of statins: a critical appraisal in the management of cancer. Pharmacol Rev 64: 102-146.

Ghosh-Choudhury N, Mandal CC, Ghosh-Choudhury N, Ghosh Choudhury G (2010) Simvastatin induces derepression of PTEN expression via NFKB to inhibit breast cancer cell growth. Cell Signal 22: 749-758.

Glynn RJ, Schneeweiss S, Wang PS, Levin R, Avorn J (2006) Selective prescribing led to overestimation of the benefits of lipid-lowering drugs. J Clin Epidemiol 59: 819-828.

Gustbée E, Tryggvadottir H, Markkula A, Simonsson M, Nodin B, Jirström K, Rose C, Ingvar C, Borgquist S, Jernström H (2015) Tumor-specific expression of HMG-CoA reductase in a population-based cohort of breast cancer patients. BMC Clin Pathol 15: 8 .
Hillner BE, Penberthy L, Desch CE, McDonald MK, Smith TJ, Retchin SM (1996) Variation in staging and treatment of local and regional breast cancer in the elderly. Breast Cancer Res Treat 40: 75-86.

Holmes MD, Chen WY (2012) Hiding in plain view: the potential for commonly used drugs to reduce breast cancer mortality. Breast Cancer Res BCR 14: 216

Holmes MD, Chen WY, Li L, Hertzmark E, Spiegelman D, Hankinson SE (2010) Aspirin intake and survival after breast cancer. J Clin Oncol Off J Am Soc Clin Oncol 28: 1467-1472.

Howlader N, Ries LAG, Mariotto AB, Reichman ME, Ruhl J, Cronin KA (2010) Improved estimates of cancer-specific survival rates from population-based data. J Natl Cancer Inst 102: 1584-1598.

Jakobisiak M, Golab J (2003) Potential antitumor effects of statins (Review). Int J Oncol 23: 1055-1069.

Kimbung S, Lettiero B, Feldt M, Bosch A, Borgquist S, Kimbung S, Lettiero B, Feldt M, Bosch A, Borgquist S (2016) High expression of cholesterol biosynthesis genes is associated with resistance to statin treatment and inferior survival in breast cancer. Oncotarget 5: 59640-59651.

Kwan ML, Habel LA, Flick ED, Quesenberry CP, Caan B (2008) Post-diagnosis statin use and breast cancer recurrence in a prospective cohort study of early stage breast cancer survivors. Breast Cancer Res Treat 109: $573-579$.

Mansourian M, Haghjooy-Javanmard S, Eshraghi A, Vaseghi G, Hayatshahi A, Thomas J (2016) Statins use and risk of breast cancer recurrence and death: a systematic review and meta-analysis of observational studies. J Pharm Pharm Sci 19: 72-81.

Manthravadi S, Shrestha A, Madhusudhana S (2016) Impact of statin use on cancer recurrence and mortality in breast cancer: a systematic review and meta-analysis. Int J Cancer 139(6): 1281-1288.

Marshall SF, Bernstein L, Anton-Culver H, Deapen D, Horn-Ross PL, Mohrenweiser H, Peel D, Pinder R, Purdie DM, Reynolds P, Stram D, West D, Wright WE, Ziogas A, Ross RK (2005) Nonsteroidal anti-inflammatory drug use and breast cancer risk by stage and hormone receptor status. J Natl Cancer Inst 97: 805-812.

Matusewicz L, Meissner J, Toporkiewicz M, Sikorski AF (2015) The effect of statins on cancer cells-review. Tumour Biol J Int Soc Oncodevelopmental Biol Med 36: 4889-4904.

Mc Menamin ÚC, Murray LJ, Hughes CM, Cardwell CR (2016) Statin use and breast cancer survival: a nationwide cohort study in Scotland. BMC Cancer 16: 600 .

McDonnell DP, Chang C-Y, Nelson ER (2014) The estrogen receptor as a mediator of the pathological actions of cholesterol in breast cancer. Climacteric J Int Menopause Soc 17(Suppl 2): 60-65.

Mira E, Carmona-Rodríguez L, Tardáguila M, Azcoitia I, González-Martín A, Almonacid L, Casas J, Fabriás G, Mañes S, Mira E, Carmona-Rodríguez L, Tardáguila M, Azcoitia I, González-Martín A, Almonacid L, Casas J, Fabriás G, Mañes S (2013) A lovastatin-elicited genetic program inhibits M2 macrophage polarization and enhances $\mathrm{T}$ cell infiltration into spontaneous mouse mammary tumors. Oncotarget 4: 2288-2301.

Murtola TJ, Visvanathan K, Artama M, Vainio H, Pukkala E (2014) Statin use and breast cancer survival: a nationwide cohort study from Finland. PLoS ONE 9: e110231.

Nickels S, Vrieling A, Seibold P, Heinz J, Obi N, Flesch-Janys D, Chang-Claude J (2013) Mortality and recurrence risk in relation to the use of lipid-lowering drugs in a prospective breast cancer patient cohort. PLoS ONE 8: e75088.

Peterson AM, Nau DP, Cramer JA, Benner J, Gwadry-Sridhar F, Nichol M (2007) A checklist for medication compliance and persistence studies using retrospective databases. Value Health J Int Soc Pharmacoeconomics Outcomes Res 10: 3-12.

Robinson JG, Booth B (2010) Statin use and lipid levels in older adults: NHANES 2001-2006. J Clin Lipidol 4: 483-490.

Schneeweiss S, Seeger JD, Maclure M, Wang PS, Avorn J, Glynn RJ (2001) Performance of comorbidity scores to control for confounding in epidemiologic studies using claims data. Am J Epidemiol 154: 854-864.

Smith A, Murphy L, Bennett K, Barron TI (2017) Patterns of statin initiation and continuation in patients with breast or colorectal cancer, towards end-of-life. Support Care Cancer Off J Multinatl Assoc Support Care Cancer 25(5): 1629-1637.

Smith A, Murphy L, Sharp L, O'Connor D, Gallagher WM, Bennett K, Barron TI (2016) De novo post-diagnosis statin use, breast cancer-specific 
and overall mortality in women with stage I-III breast cancer. $\mathrm{Br} J$ Cancer 115(5): 592-598.

Tchrakian N, Flanagan L, Harford J, Gannon JM, Quinn CM (2015) New ASCO/CAP guideline recommendations for HER2 testing increase the proportion of reflex in situ hybridization tests and of HER2 positive breast cancers. Virchows Arch 468: 207-211.

Tobert JA (2003) Lovastatin and beyond: the history of the HMG-CoA reductase inhibitors. Nat Rev Drug Discov 2: 517-526.

Walley T, Folino-Gallo P, Schwabe U, van Ganse E (2004) Variations and increase in use of statins across Europe: data from administrative databases. BMJ 328: 385-386.
WHOCC-ATC/DDD Index http://www.whocc.no/atc_ddd_index/(accessed 09 Feb 2015).

Zhong S, Zhang X, Chen L, Ma T, Tang J, Zhao J (2015) Statin use and mortality in cancer patients: Systematic review and meta-analysis of observational studies. Cancer Treat Rev 41(6): 554-567.

This work is published under the standard license to publish agreement. After 12 months the work will become freely available and the license terms will switch to a Creative Commons AttributionNonCommercial-Share Alike 4.0 Unported License.

Supplementary Information accompanies this paper on British Journal of Cancer website (http://www.nature.com/bjc) 